\title{
Early Detection of Tumor Response by FLT/MicroPET Imaging in a C26 Murine Colon Carcinoma Solid Tumor Animal Model
}

\author{
Wan-Chi Lee, ${ }^{1}$ Chih-Hsien Chang, ${ }^{1,2}$ Chung-Li Ho, ${ }^{1}$ Liang-Cheng Chen, ${ }^{1}$ Yu-Hsien Wu, ${ }^{1}$ \\ Jenn-Tzong Chen, ${ }^{1}$ Ying-Ling Wang, ${ }^{1}$ and Te-Wei Lee ${ }^{1}$ \\ ${ }^{1}$ Isotope Application Division, Institute of Nuclear Energy Research, Taoyuan 32546, Taiwan \\ ${ }^{2}$ Department of Biomedical Imaging and Radiological Sciences, National Yang-Ming University, Taipei 11221, Taiwan
}

Correspondence should be addressed to Te-Wei Lee, twlee@iner.gov.tw

Received 21 April 2011; Revised 22 June 2011; Accepted 27 June 2011

Academic Editor: David J. Yang

Copyright () 2011 Wan-Chi Lee et al. This is an open access article distributed under the Creative Commons Attribution License, which permits unrestricted use, distribution, and reproduction in any medium, provided the original work is properly cited.

\begin{abstract}
Fluorine-18 fluorodeoxyglucose $\left({ }^{18} \mathrm{~F}-\mathrm{FDG}\right)$ positron emission tomography (PET) imaging demonstrated the change of glucose consumption of tumor cells, but problems with specificity and difficulties in early detection of tumor response to chemotherapy have led to the development of new PET tracers. Fluorine-18-fluorothymidine $\left({ }^{18} \mathrm{~F}\right.$-FLT) images cellular proliferation by entering the salvage pathway of DNA synthesis. In this study, we evaluate the early response of colon carcinoma to the chemotherapeutic drug, lipo-Dox, in C26 murine colorectal carcinoma-bearing mice by ${ }^{18} \mathrm{~F}-\mathrm{FDG}$ and ${ }^{18} \mathrm{~F}$-FLT. The male BALB/c mice were bilaterally inoculated with $1 \times 10^{5}$ and $1 \times 10^{6} \mathrm{C} 26$ tumor cells per flank. Mice were intravenously treated with $10 \mathrm{mg} / \mathrm{kg}$ lipo-Dox at day 8 after ${ }^{18} \mathrm{~F}$-FDG and ${ }^{18} \mathrm{~F}$-FLT imaging. The biodistribution of ${ }^{18} \mathrm{~F}$-FDG and ${ }^{18} \mathrm{~F}$-FLT were followed by the microPET imaging at day 9. For the quantitative measurement of microPET imaging at day $9,{ }^{18} \mathrm{~F}$-FLT was superior to ${ }^{18} \mathrm{~F}$-FDG for early detection of tumor response to Lipo-DOX at various tumor sizes $(P<0.05)$. The data of biodistribution showed similar results with those from the quantification of SUV (standard uptake value) by microPET imaging. The study indicates that ${ }^{18} \mathrm{~F}-\mathrm{FLT} / \mathrm{microPET}$ is a useful imaging modality for early detection of chemotherapy in the colorectal mouse model.
\end{abstract}

\section{Introduction}

${ }^{18} \mathrm{~F}$-fluorodeoxyglucose $\left({ }^{18} \mathrm{~F}-\mathrm{FDG}\right)$ is widely used as a noninvasive marker in clinical oncology. ${ }^{18} \mathrm{~F}-\mathrm{FDG}$, an analog of glucose, undergoes phosphorylation by hexokinase, cannot further enter glycolysis, and is then trapped in the cell. ${ }^{18} \mathrm{~F}-$ FDG has high-detection sensitivity, but not specificity; the property showed the difficulty of distinguishing proliferation tumor cells from inflammatory cells, making it less suitable as a marker for studying drug response $[1-3]$. Because ${ }^{18} \mathrm{~F}-$ FDG is also metabolized in nontumor tissues, the results may be false-positive in inflammation or reactive tissue $[4,5]$. For these reasons, ${ }^{18} \mathrm{~F}$-FDG is not suitable as a marker for early response to treatment of cancer. Another approach for tumor visualization is using radiolabeled nucleosides such as ${ }^{11} \mathrm{C}$-thymidine. Since ${ }^{11} \mathrm{C}$-thymidine is rapidly incorporated into newly synthesized DNA, this radiopharmaceutical can be used to image cellular proliferation [3]. However, the halflife of ${ }^{11} \mathrm{C}$-thymidine and the poor imaging quality limit its clinical application. These limitations led to the development of analogues that are more resistant to degradation and can be labeled with radionuclides more conductive to routine clinical use, such as ${ }^{18} \mathrm{~F}[6]$.

The trapping of ${ }^{18} \mathrm{~F}$-FLT demonstrated with the uptake of thymidine analogue after phosphorylation by thymidine kinase 1 (TK1) in the S phase of cell cycle [7]. Shields et al. used ${ }^{18} \mathrm{~F}$-FLT for noninvasive PET imaging because ${ }^{18} \mathrm{~F}$ FLT is an analog of thymidine, and the half-life of ${ }^{18} \mathrm{~F}$ is longer than that of ${ }^{11} \mathrm{C}[8] .{ }^{18} \mathrm{~F}-\mathrm{FLT}$ was phosphorylated and incorporated into DNA, which is equivalent to cell proliferation. DNA production slows as the cell division decreases, which is one of the earliest responses to cancer therapy $[6,9]$. Because DNA synthesis associated with cell proliferation turns off faster than glucose utilization, ${ }^{18} \mathrm{~F}$ FLT offers a more rapid measure of response than ${ }^{18} \mathrm{~F}-$ FDG. ${ }^{18}$ F-FLT has been found to be useful for noninvasively assessing the proliferation rate of several types of tumor, such as colorectal, oesophageal, and lung tumor [10-13]. 
The ${ }^{18}$ F-FLT could outperform ${ }^{18} \mathrm{~F}$-FDG for monitoring the response to cytostatic chemotherapy $[9,14]$. Evaluation of tumor proliferative activity by PET using ${ }^{18} \mathrm{~F}$-FLT is a potential new procedure to assess the viability of tumors as well as the early effect of cancer therapy [15]. In addition, ${ }^{18} \mathrm{~F}$-FLT has been used to monitor the early response of tumor with chemotherapy, radiotherapy, and hormone therapy [3, 15-18].

Doxorubicin is an anthracycline antibiotic with antineoplastic activity. It is commonly used in treating a wide range of cancers. Doxorubicin can induce some side effects including nausea, vomiting, and heart arrhythmias. LipoDox is a liposome-encapsulated dosage form of doxorubicin. Its main benefit is reduced cardiotoxicity. Taking the advantage of liposome, lipo-Dox can achieve delivery from the vessel to the tumor. It makes the lipo-Dox accumulate in the tumor to increase the curative effect [19]. Our previous report also proved that lipo-Dox could efficiently inhibit the growth of C26 colorectal tumor in mice [20]. Although previous studies have demonstrated that ${ }^{18} \mathrm{~F}$-FLT might be a good alternative for followup of the tumor during/after (chemo-) radiation [5], the evaluation at various tumor sizes after chemotherapy by lipo-Dox has not been reported. This study evaluates the imaging potential of ${ }^{18} \mathrm{~F}-\mathrm{FDG}$ and ${ }^{18} \mathrm{~F}-$ FLT for early detection of tumor response to lipo-Dox in C26 colorectal tumor-bearing mice and proves that ${ }^{18} \mathrm{~F}$-FLT is better than ${ }^{18} \mathrm{~F}-\mathrm{FDG}$ in the evaluation of tumor response to chemotherapy.

\section{Materials and Methods}

2.1. Preparation of ${ }^{18} F$-FLT. The synthetic method of ${ }^{18} \mathrm{~F}$-FLT in this experiment is based on the method described by Leyton et al. [2] with the 2,3'-anhydro-5'$\mathrm{O}$-(4,4'-dimethoxytrityl)-thymidine precursor (ABX, Radeberg, Germany). Fluoride- 18 was produced from the $30 \mathrm{MeV}$ cyclotron (EBCO, Vancouver, BC, Canada) by irradiating the pneumatic liquid target at the Institute of Nuclear Energy Research (INER) and was purified by AG1x8 resin (BioRad, Hercules, Calif, USA) with $3.5 \mathrm{mg} \mathrm{K}_{2} \mathrm{CO}_{3}$ in $1 \mathrm{~mL} \mathrm{H}_{2} \mathrm{O}$ for elution before radiosynthesis. Radiofluoridation of the precursor took place in an autosynthesizer (Nuclear Interface GE, Münster, Germany) at $131^{\circ} \mathrm{C}$ and $15 \mathrm{~min}$ in DMSO with the phase transfer catalyst Kryptofix-222. Deprotection reaction of the radiofluorinated precursor was then achieved at $50^{\circ} \mathrm{C}$ for 2 min after adding $1 \mathrm{~mL}, 0.1 \mathrm{~N} \mathrm{NaOH}$. The crude product was deionized by passing through an enhanced alumina-N column (Waters, Milford, Mass, USA) and then purified by a C-18 preparative HPLC column (MachereyNagel, Düren, Germany) with a precolumn in front of it (Macherey-Nagel, Düren, Germany). The final product was sterilized by flowing through a $0.2 \mu \mathrm{m}$ membrane filter (Millipore, Billerica, Mass, USA). The radiochemical purity was analyzed by an analytical-grade HPLC system with a C-18 column (Micro Solv, Eatontown, NJ, USA). The radiochemical purity was more than $95 \%$, and the radiochemical yield was between 5 to $10 \%$.
2.2. Cell Culture, Animal Model, and Drug Administration. The C26, a murine colorectal carcinoma, was obtained from the Bioresource Collection and Research Center (Taiwan). Cells were grown in a $5 \% \mathrm{CO}_{2}$ incubator at $37^{\circ} \mathrm{C}$ in RPMI1640 medium supplemented with $10 \%$ heat-inactivated fetal bovine serum (all from Life Technologies, Gaithersburg, Md, USA). For animal inoculation, $6 \sim 8$ weeks old male BALB/c mice were obtained from the National Animal Center of Taiwan (Taipei, Taiwan) and maintained on a standard diet (Lab diet; PMI Feeds, St. Louis, Mo, USA) at room temperature, with free access to tap water in the animal house of the Institute of Nuclear Energy Research (INER), Taiwan. The mice were subcutaneously injected with $1 \times 10^{5}$ and $1 \times 10^{6}$ C26 cells in the left and right thigh, respectively, at day 0 . Tumor-bearing mice were intravenously treated with $10 \mathrm{mg} / \mathrm{kg}$ lipo-Dox (TTY) when the tumor size of $1 \times 10^{6}$ cells inoculation reached $\sim 50 \mathrm{~mm}^{3}$. At the same time, tumorbearing mice were randomized into two groups, ${ }^{18} \mathrm{~F}-\mathrm{FDG}$ and ${ }^{18} \mathrm{~F}$-FLT. The tumor size and body weight were recorded twice a week. The animal experiments were approved by the Institutional Animal Care and Use Committee (IACUC) of the INER.

2.3. Micropet Imaging and Data Analysis. The preparation of ${ }^{18} \mathrm{~F}$-FLT and ${ }^{18} \mathrm{~F}$-FDG was synthesized with an automatic apparatus at INER. MicroPET imaging was performed on the microPET-R4 system (Concorde Microsystems, Knoxville, Tenn, USA), which has a field of view of $8 \mathrm{~cm}$ axially by $11 \mathrm{~cm}$ transaxially and is capable of a spatial resolution of $2.3 \mathrm{~mm}$ and an absolute sensitivity of $1020 \mathrm{cps} / \mu \mathrm{Ci}$ in the middle of the field of view. To monitor the progression of the tumors, microPET scanning was performed with ten mice (five mice per each group) on days 4, 8, and 9 after tumor inoculation. Static microPET images were acquired $60 \mathrm{~min}$ after i.v. administration of $\sim 200 \mu \mathrm{Ci}$ of ${ }^{18} \mathrm{~F}-\mathrm{FDG}$ or ${ }^{18} \mathrm{~F}$-FLT to evaluate the capability of radiopharmaceuticals to differentiate the tumor uptake subsequent to chemotherapy. The image list-mode data were reconstructed to one frame. Fourier rebinning and 2D filtered back-projection reconstruction using Ramp filters, with one half of the Nyquist frequency as the cut-off frequency, were applied to all static data. For data analysis, a region of interest (ROI) was placed on each tumor and muscle along the spine in the transaxial images, including the entire lesion volume. The average radioactivity concentration within a tumor or muscle region was obtained from the average pixel value within the multiple ROI volumes. AsiPro software (Concorde Microsystems, Knoxville, Tenn, USA) was used to view the microPET images and for data analysis. The counts in each ROI were converted to radioactivity per gram of tissue ( $\mathrm{nCi} / \mathrm{g}$ ), assuming a tissue density of $1 \mathrm{~g} / \mathrm{mL}$, and then normalized to a percentage injected dose per gram of tissues $(\% \mathrm{ID} / \mathrm{g})[21,22]$. The $\% \mathrm{ID} / \mathrm{g}$ was defined as the average radioactivity concentration in several planes of the tumor or organs divided by the total injected activity. The standard uptake value, SUV, was calculated as follows: $\mathrm{SUV}=(\mathrm{A} \times$ W)/Ainj, where A is the activity of ROI, W is the body 
weight of the mice, and Ainj is the injection dose of the radiopharmaceutical.

2.4. Biodistribution of ${ }^{18}$ F-FDG and ${ }^{18} F-F L T$. Biodistribution studies of the ${ }^{18} \mathrm{~F}$-FDG and ${ }^{18} \mathrm{~F}$-FLT were performed in $\mathrm{BALB} / \mathrm{c}$ mice bearing $\mathrm{C} 26$ tumors. The mice were intravenously injected with $\sim 30 \mu \mathrm{Ci}$ of ${ }^{18} \mathrm{~F}$-FLT or ${ }^{18} \mathrm{~F}$-FDG at days 4,8 , and 9 after inoculation of tumor cells. Five mice at each time point were euthanized by $\mathrm{CO}_{2}$ administration 60 mins after the injection. Blood samples were collected through cardiac puncture after euthanizing the mice. The organs of interest were removed, washed, and weighed, and the radioactivity was measured with a gamma counter (1470 WIZARD Gamma Counter, Wallac, Finland). The standards $(n=5)$ were prepared to verify the counting efficiency of the gamma counter for each radiotracer. The $\% \mathrm{ID} / \mathrm{g}$ values were calculated by comparison with standards representing the injected dose per animal. Data were expressed as mean \pm standard deviation $(n=5)$.

2.5. Statistical Method. Unpaired $t$-tests were performed to compare the differences between the data. Differences were considered significant at the $95 \%$ confidence level $(P<0.05)$.

\section{Results}

3.1. Longitudinal Tumor Imaging with ${ }^{18} F-F D G$ or ${ }^{18} F-F L T$. The uptake of ${ }^{18} \mathrm{~F}$-FDG $/{ }^{18} \mathrm{~F}$-FLT in the tumor and muscle was determined longitudinally by the microPET images from day 4 after tumor inoculation, corresponding to day 4, day 8, and day 9 after inoculation of C26 murine colon carcinoma cells (Figure 1). At day 8, after the microPET imaging was obtained, mice were injected with $10 \mathrm{mg} / \mathrm{kg}$ lipo-Dox intravenously. The uptake of ${ }^{18} \mathrm{~F}-\mathrm{FDG}$ in the tumor increased following the growth of the tumor from day 4 to day 9 after inoculation of the murine colon carcinoma. For the uptake of ${ }^{18} \mathrm{~F}$-FLT, the accumulation of ${ }^{18} \mathrm{~F}$-FLT reached a plateau at day 8 and decreased at day 9 , when $10 \mathrm{mg} / \mathrm{kg}$ lipoDox was administered. ${ }^{18} \mathrm{~F}-\mathrm{FDG} / \mathrm{PET}$ imaging showed high accumulation in the heart, abdomen, and bladder. The ${ }^{18} \mathrm{~F}-$ FLT/PET imaging showed lower background accumulation in the heart than ${ }^{18} \mathrm{~F}-\mathrm{FDG} / \mathrm{PET}$ imaging. The accumulations of ${ }^{18} \mathrm{~F}-\mathrm{FDG}$ in tumors were $4.05 \pm 0.50 \% \mathrm{ID} / \mathrm{g}$ and $4.13 \pm$ $0.49 \% \mathrm{ID} / \mathrm{g}$ at day 8 and day 9 after inoculation of $10^{5}$ colon carcinoma cells and in tumors were $4.23 \pm 0.41 \% \mathrm{ID} / \mathrm{g}$ and $4.47 \pm 0.29 \% \mathrm{ID} / \mathrm{g}$ at day 8 and day 9 after inoculation of $10^{6}$ colon carcinoma cells. The accumulations of ${ }^{18} \mathrm{~F}$-FLT in tumors were $1.62 \pm 0.11 \% \mathrm{ID} / \mathrm{g}$ and $1.23 \pm 0.03 \% \mathrm{ID} / \mathrm{g}$ at day 8 and day 9 after inoculation of $10^{5}$ colon carcinoma cells and in tumors were $2.04 \pm 0.13 \% \mathrm{ID} / \mathrm{g}$ and $1.34 \pm 0.03 \% \mathrm{ID} / \mathrm{g}$ at day 8 and day 9 after inoculation of $10^{6}$ colon carcinoma cells. (Figure 2). The uptake of ${ }^{18} \mathrm{~F}$-FLT was significantly decreased at day 9 compared with that at day $8(P<0.05)$.

${ }^{18} \mathrm{~F}$-FDG showed tumor-to-muscle (T/M) ratios were 2.08 and 2.19 at day 8 , which did not significantly differ from its $\mathrm{T} / \mathrm{M}$ ratios 2.08 and 2.27 at day 9 after inoculation of $10^{5}$ and $10^{6}$ tumor cells at Day 0 , respectively. In contrast, the $\mathrm{T} / \mathrm{M}$ ratios of ${ }^{18} \mathrm{~F}$-FLT were 1.44 and 1.79 at day 8 , which were both significantly different from its $\mathrm{T} / \mathrm{M}$ ratio 1.29 and 1.40 at day $9(P<0.05)$ after inoculation of $10^{5}$ and $10^{6}$ tumor cells at Day 0 , respectively. These results demonstrated that ${ }^{18} \mathrm{~F}$-FLT/PET could decrease the radioactivity accumulation in C26 colon carcinoma-bearing animals.

3.2. Biodistribution of ${ }^{18} F$-FLT and ${ }^{18} F$-FDG. The biodistribution data of ${ }^{18} \mathrm{~F}-\mathrm{FLT}$ and ${ }^{18} \mathrm{~F}-\mathrm{FDG}$ at day 4, day 8 , and day 9 following tumor inoculation in C26-bearing mice were shown in Table 1 . The results of ${ }^{18} \mathrm{~F}$-FDG showed significant accumulation in the brain, red marrow, heart, and tumor. The radioactivity of ${ }^{18} \mathrm{~F}-\mathrm{FDG}$ in most organs was not significantly different, whether at day 4 , day 8 , or day 9 . The $\mathrm{T} / \mathrm{M}$ ratio of ${ }^{18} \mathrm{~F}-\mathrm{FDG}$ in $10^{5}$ and $10^{6}$ had the same profile and slightly decreased from day 4 to day 9 . The results of ${ }^{18} \mathrm{~F}$-FLT showed significant accumulation in the red marrow, kidney, bladder, small intestine, and tumors. The radioactivity of ${ }^{18} \mathrm{~F}-$ FLT in most organs significantly differed at day 4, day 8 , and day 9. The biodistribution data showed that the organs increased from day 4 to day 8 and decreased from day 8 to day 9 after lipo-Dox treatment. The T/M ratio of ${ }^{18} \mathrm{~F}$ FLT after $10^{5}$ and $10^{6}$ tumor cells inoculated had the same profile, slightly decreasing from day 4 to day 9. According to biodistribution of ${ }^{18} \mathrm{~F}$-FLT, there were significant differences from its T/M ratio at day 8 and day 9 , both in $10^{5}(P<0.05)$ and $10^{6}(P<0.01)$ tumor cell inoculation. However, it was not represented in ${ }^{18}$ F-FDG (Figure 3).

\section{Discussion}

${ }^{18} \mathrm{~F}-\mathrm{FLT}$ is trapped within the cytosol after being monophosphorylated by TK1, a principal enzyme in the salvage pathway [7]. The advantages of radiolabeled thymidine are (1) it can be quickly absorbed by the cell; (2) it is stable when it is phosphorylated by TK1, (3) it can be incorporated into the DNA, so it becomes metabolically trapped $[12,23]$. The activity of TK1 is known to be high in proliferating and malignant cells and low or absent in quiescent cells. The potential of ${ }^{18} \mathrm{~F}$-FLT/PET to measure early cytostasis and cytotoxicity induced by the chemotherapy drug, cisplatin, in RIF-1-bearing mice has been assessed [2]. An early response to therapy using ${ }^{18} \mathrm{~F}$-FLT in gastric cancer has also been found [24]. It was reported that the surviving fraction of SCCVII cells determined by clonogenic assay was $80 \%$ after $10 \mathrm{~Gy}$ of irradiation, despite a marked reduction in the ${ }^{18} \mathrm{~F}-$ FLTuptake to the background level at $24 \mathrm{~h}$ after a single irradiation session [25]. Murayama et al. also demonstrated the results supported their interpretation that the reduction of ${ }^{18}$ F-FLT uptake represented cells showing biologic impairment of proliferation by irradiation after $24 \mathrm{hr}$ [26]. Dimitrakopoulou-Strauss and Strauss indicated that the role of ${ }^{18} \mathrm{~F}$-FLT is not to stage a tumor, but to biologically characterize a tumor process and to assess the potential impact of ${ }^{18} \mathrm{~F}$-FLT uptake with respect to prognosis and therapeutic outcome [27]. These results showed that ${ }^{18} \mathrm{~F}$-FLT could be an index to monitor the proliferation of colorectal carcinoma after chemotherapy. Earlier information on lack of chemosensitivity will enable oncologist to stop treatment 

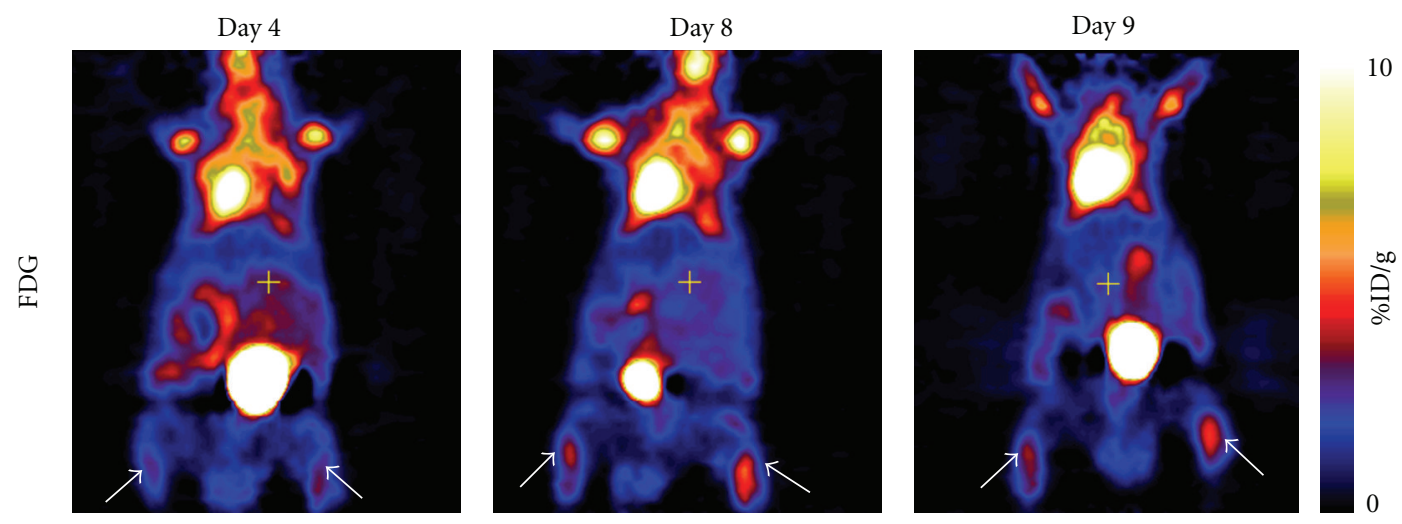

(a)
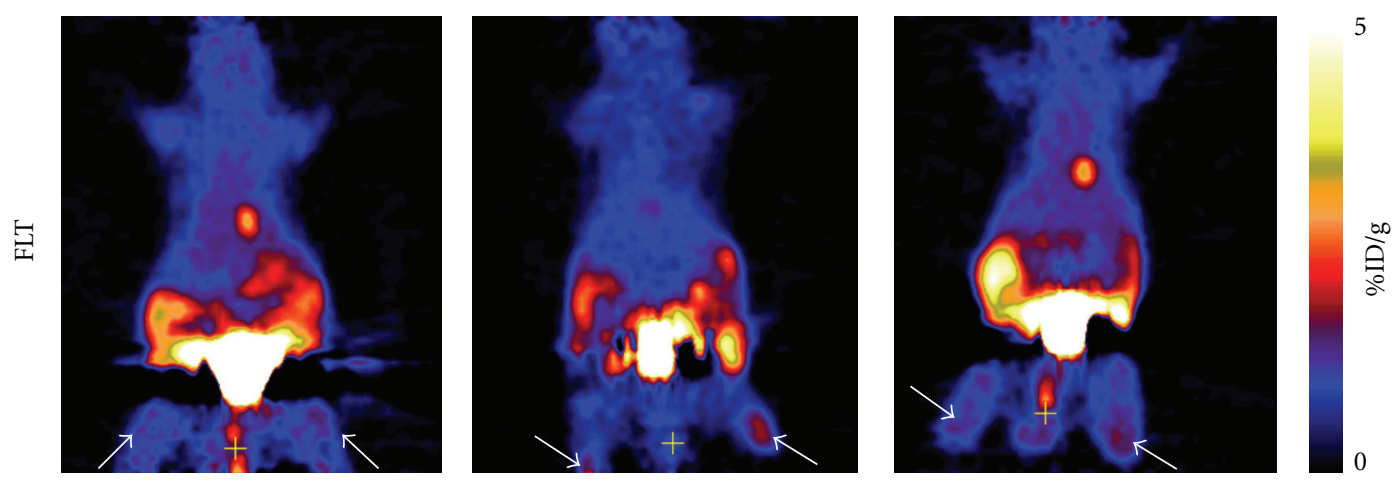

(b)

FIGURE 1: Coronal microPET image showed the radioactivity of ${ }^{18} \mathrm{~F}-\mathrm{FDG}$ (a) and ${ }^{18} \mathrm{~F}$-FLT (b) at days 4,8 , and 9 after tumor inoculation. ${ }^{18} \mathrm{~F}$-FLT of microPET imaging were presented less activity of background than ${ }^{18} \mathrm{~F}$-FDG. The tumor-bearing mice were administrated with Lipo-Dox at day 8 after tumor inoculation. (Arrow indicated the C26 tumor; the right side was $10^{6}$ tumor cell inoculation, and the left side was $10^{5}$ cell inoculation.)

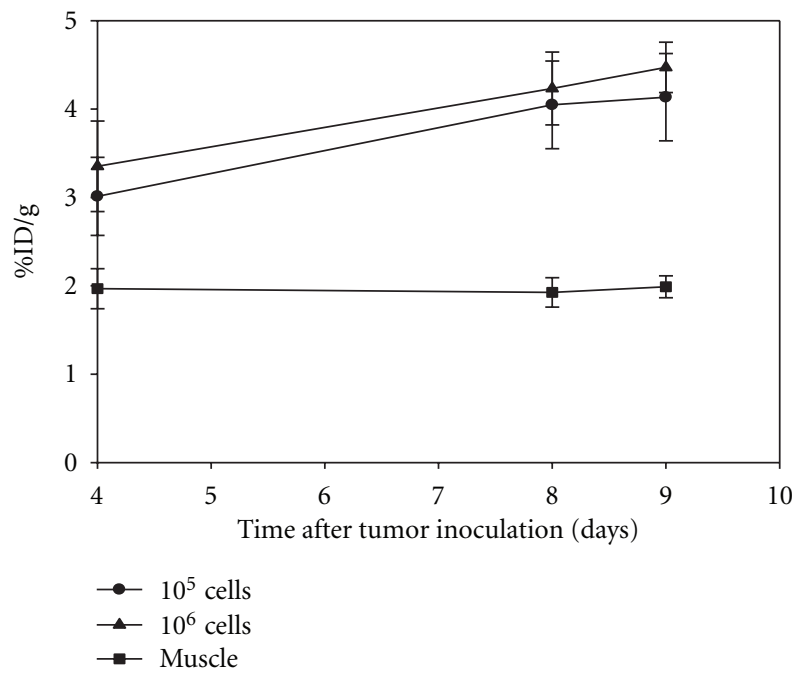

(a)

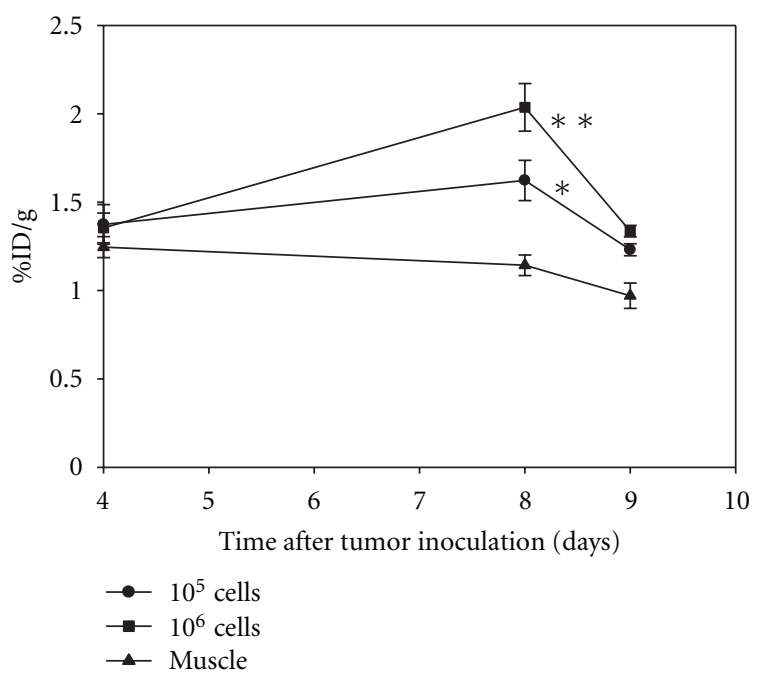

(b)

FIGURE 2: Longitudinal quantification of the progression of tumor growth in various sizes in C26-bearing mice derived from ${ }^{18} \mathrm{~F}$-FDG (a) or ${ }^{18} \mathrm{~F}$-FLT (b) microPET images. The uptakes of SUV in ${ }^{18} \mathrm{~F}$-FLT were significantly decreased after lipo-Dox treatment. (mean $\pm \mathrm{SD}, n=3$; ${ }^{*} P$ $<0.05$; $\left.{ }^{* *} P<0.01\right)$. 
TABLE 1: Biodistribution of ${ }^{18} \mathrm{~F}-\mathrm{FDG} /{ }^{18} \mathrm{~F}-\mathrm{FLT}$ after i.v. injection.

\begin{tabular}{|c|c|c|c|c|c|c|}
\hline \multirow[b]{2}{*}{ Organ } & \multicolumn{3}{|c|}{ FDG } & \multicolumn{3}{|c|}{ FLT } \\
\hline & Day 4 & Day 8 & Day 9 & Day 4 & Day 8 & Day 9 \\
\hline Whole blood & $0.39 \pm 0.01$ & $0.42 \pm 0.04$ & $0.46 \pm 0.03$ & $1.04 \pm 0.19$ & $0.76 \pm 0.03$ & $1.24 \pm 0.20$ \\
\hline Brain & $6.93 \pm 0.49$ & $6.70 \pm 0.60$ & $5.44 \pm 0.52$ & $0.21 \pm 0.03$ & $0.15 \pm 0.01$ & $0.22 \pm 0.03$ \\
\hline Skin & $1.11 \pm 0.06$ & $1.12 \pm 0.10$ & $0.79 \pm 0.17$ & $0.66 \pm 0.13$ & $0.55 \pm 0.04$ & $0.74 \pm 0.12$ \\
\hline Muscle & $2.62 \pm 0.41$ & $2.27 \pm 0.27$ & $2.78 \pm 0.64$ & $0.75 \pm 0.22$ & $0.68 \pm 0.08$ & $1.01 \pm 0.21$ \\
\hline Bone & $1.71 \pm 0.23$ & $1.66 \pm 0.23$ & $1.93 \pm 0.32$ & $0.93 \pm 0.08$ & $0.93 \pm 0.15$ & $0.50 \pm 0.06$ \\
\hline Red marrow & $11.89 \pm 6.11$ & $8.67 \pm 0.97$ & $5.54 \pm 1.00$ & $1.77 \pm 0.65$ & $3.01 \pm 1.08$ & $0.98 \pm 0.30$ \\
\hline Heart & $15.55 \pm 1.33$ & $16.94 \pm 1.95$ & $20.95 \pm 2.34$ & $0.80 \pm 0.15$ & $0.67 \pm 0.06$ & $0.96 \pm 0.18$ \\
\hline Lung & $2.26 \pm 0.11$ & $2.46 \pm 0.21$ & $2.40 \pm 0.22$ & $0.81 \pm 0.15$ & $0.64 \pm 0.03$ & $0.95 \pm 0.13$ \\
\hline Testis & $2.44 \pm 0.10$ & $2.77 \pm 0.20$ & $2.23 \pm 0.18$ & $1.49 \pm 0.14$ & $1.25 \pm 0.09$ & $1.71 \pm 0.19$ \\
\hline Spleen & $2.71 \pm 0.31$ & $3.08 \pm 0.18$ & $3.38 \pm 0.16$ & $1.79 \pm 0.29$ & $2.26 \pm 0.23$ & $1.23 \pm 0.21$ \\
\hline Pancreas & $1.73 \pm 0.09$ & $1.83 \pm 0.13$ & $2.14 \pm 0.11$ & $0.98 \pm 0.19$ & $0.76 \pm 0.04$ & $1.18 \pm 0.17$ \\
\hline Kidney & $2.01 \pm 0.24$ & $1.33 \pm 0.15$ & $1.90 \pm 0.15$ & $2.27 \pm 0.29$ & $2.46 \pm 0.27$ & $3.07 \pm 0.62$ \\
\hline Adrenals & $3.87 \pm 0.67$ & $4.55 \pm 0.43$ & $4.32 \pm 0.59$ & $1.44 \pm 0.22$ & $2.48 \pm 0.62$ & $1.69 \pm 0.44$ \\
\hline Liver & $0.80 \pm 0.03$ & $0.96 \pm 0.05$ & $0.93 \pm 0.05$ & $1.03 \pm 0.16$ & $0.80 \pm 0.05$ & $1.17 \pm 0.18$ \\
\hline Bladder & $2.63 \pm 0.56$ & $2.13 \pm 0.32$ & $2.75 \pm 0.86$ & $3.42 \pm 0.33$ & $4.84 \pm 0.77$ & $3.81 \pm 0.55$ \\
\hline Stomach & $3.25 \pm 0.27$ & $5.07 \pm 0.47$ & $4.54 \pm 0.49$ & $0.87 \pm 0.13$ & $0.81 \pm 0.05$ & $1.06 \pm 0.22$ \\
\hline Small intestine & $3.04 \pm 0.28$ & $5.33 \pm 1.59$ & $3.86 \pm 0.70$ & $3.19 \pm 0.73$ & $3.28 \pm 0.71$ & $2.31 \pm 0.25$ \\
\hline Large intestine & $7.11 \pm 0.61$ & $7.81 \pm 0.85$ & $7.29 \pm 0.73$ & $1.54 \pm 0.22$ & $1.76 \pm 0.40$ & $2.27 \pm 0.31$ \\
\hline $10^{5}$ tumor & $4.67 \pm 1.16$ & $5.30 \pm 0.62$ & $4.32 \pm 0.56$ & $1.16 \pm 0.40$ & $2.70 \pm 0.34$ & $2.12 \pm 0.24$ \\
\hline $10^{6}$ tumor & $9.03 \pm 3.58$ & $4.83 \pm 0.61$ & $3.92 \pm 0.46$ & $2.38 \pm 0.37$ & $3.27 \pm 0.13$ & $2.09 \pm 0.20$ \\
\hline $10^{5} \mathrm{~T} / \mathrm{M}$ & 1.58 & 2.62 & 1.98 & 1.65 & $4.19^{*}$ & 2.38 \\
\hline $10^{6} \mathrm{~T} / \mathrm{M}$ & 3.49 & 2.40 & 1.81 & 4.02 & $5.13^{* *}$ & 2.24 \\
\hline
\end{tabular}

Data were expressed as \%ID/g (Mean $\pm \operatorname{SEM}, n=5)\left(*: P<0.05\right.$, significantly different at day 8 and day 9 in $10^{5}$ tumor cell inoculation; $* *: P<0.05$, significantly different at day 8 and day 9 in $10^{6}$ tumor cell inoculation).

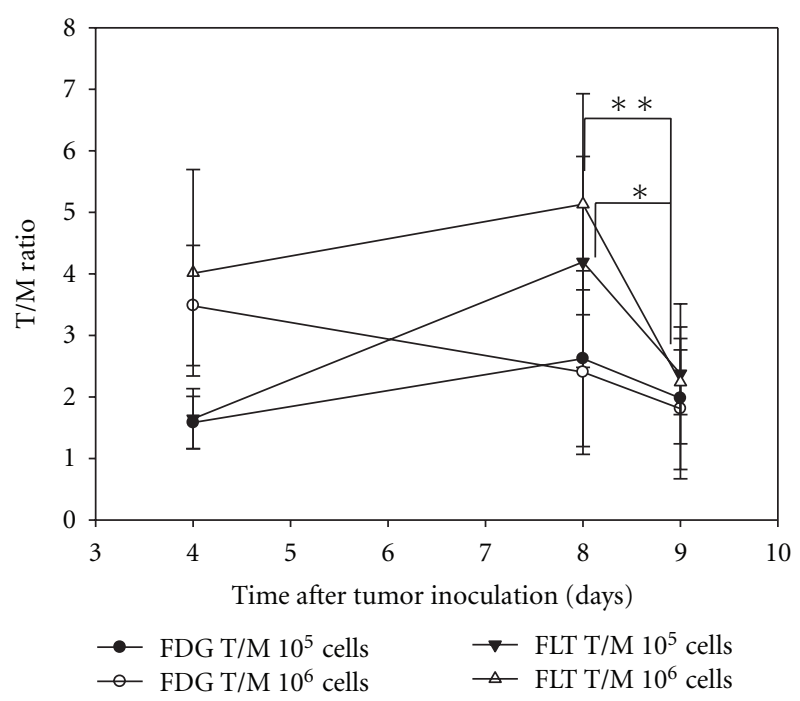

Figure 3: Tumor-to-muscle ratio of ${ }^{18} \mathrm{~F}$-FLT and ${ }^{18} \mathrm{~F}-\mathrm{FDG}$ in $10^{5}$ and $10^{6}$ tumor cells inoculation obtained from biodistribution. There were significant differences in the detection of microPET-FLT between day 8 and day 9 . (mean $\pm \mathrm{SD}, n=5 ;{ }^{*} P<0.05$; ${ }^{*} P<0.01$ ).

and prevent undue toxicity associated with treatment, as well as reduce the cost of treatment.
In this study, we utilized ${ }^{18} \mathrm{~F}$-FDG and ${ }^{18} \mathrm{~F}$-FLT/PET to monitor the early response after treatment of lipo-Dox in $\mathrm{C} 26$ tumor-bearing mice. ${ }^{18} \mathrm{~F}$-FDG uptake is related to increased glucose metabolism. Since most cancer cells are metabolically active but fewer cells are proliferating, a higher uptake of ${ }^{18} \mathrm{~F}$-FDG than of ${ }^{18} \mathrm{~F}$-FLT in the tumor can be expected [28]. Although the uptake of ${ }^{18}$ F-FLT in gastric cancer was significantly lower than that of ${ }^{18} \mathrm{~F}-\mathrm{FDG}$, the ${ }^{18} \mathrm{~F}$-FLT/PET showed as high sensitivity as ${ }^{18} \mathrm{~F}$-FDG/PET in detection of gastric cancer [29]. We tried to monitor the different size of C26 tumor model which was designed for early detection. In the study, we tried to image the microPET images 4 days after tumor inoculation, when the tumor could not be observed. Unfortunately, this information also could not be obtained clearly from the microPET imaging, regardless of whether ${ }^{18} \mathrm{~F}$-FLT or ${ }^{18} \mathrm{~F}$-FDG/PET was used. We determined the day for chemotherapy to be 8 days after tumor inoculation. Because there are many studies indicating when the tumor size is $<100 \mathrm{~mm}^{3}$, better therapeutic efficacy can be expected. In the present study, no significant changes in tumor size measured by caliper after $2 \sim 3$ days of lipo-Dox treatment. However, ${ }^{18} \mathrm{~F}$-FLT/PET can detect the regression of tumor after $24 \mathrm{hr}$ of lipo-Dox treatment. ${ }^{18} \mathrm{~F}-$ FLT is superior to ${ }^{18} \mathrm{~F}-\mathrm{FDG}$ in detecting the change in tumor size (Figure 2). Biodistribution data also showed 
the similar profile (Table 1). The tumor-to-muscle ratio identified significant difference by ${ }^{18} \mathrm{~F}-\mathrm{FLT}$ in $10^{6}$ tumors $(P=0.008 ; P<0.01) .{ }^{18}$ F-FLT/PET correlated well with cellular proliferation makers in both primary and metastasis colorectal cancer has been reported [10].

The uptake of ${ }^{18}$ F-FLT can be an index for cellular proliferation. PET imaging permits the evaluation of a tumor in its entirety, overcoming issues of heterogeneity and tumor sampling errors and can easily be reported at any point during treatment [28]. PET imaging is the most promising technique for early response monitoring, and many potential selective (e.g., ${ }^{18}$ F-FLT) and less selective (e.g., ${ }^{18}$ F-FDG) PET tracers are available [14]. Therefore, ${ }^{18} \mathrm{~F}$-FLT/PET is a potential tool for noninvasive in vivo assessment for early response after therapy. ${ }^{18}$ F-FLT showed decreased uptake with lipo-Dox treatment, suggesting that this new tracer may play a role in improving the diagnostic specificity of PET. This study showed that ${ }^{18}$ F-FLT/PET was superior to ${ }^{18} \mathrm{~F}-\mathrm{FDG} / \mathrm{PET}$ in distinguishing the early response of Lipo-Dox in the C26 tumor. We provided the tool of ${ }^{18} \mathrm{~F}$ FLT/PET as a proliferation index in C26-bearing mice. In conclusions, we suggest that ${ }^{18} \mathrm{~F}$-FLT might be a good alternative for followup of the tumor after chemotherapy. This study demonstrated that ${ }^{18} \mathrm{~F}$-FLT is a sensitive tracer for the imaging of proliferation in tumors and is also a good tracer to detect early response to chemotherapy treatment.

\section{Acknowledgment}

The authors would like to thank Dr. M. L. Jan, W. C. Hsu, T. J. Chang, and C. H. Yen for their technical assistance.

\section{References}

[1] D. A. Mankoff, J. F. Eary, J. M. Link et al., "Tumor-specific positron emission tomography imaging in patients: $\left[{ }^{18} \mathrm{~F}\right]$ fluorodeoxyglucose and beyond," Clinical Cancer Research, vol. 13, no. 12, pp. 3460-3469, 2007.

[2] J. Leyton, J. R. Latigo, M. Perumal, H. Dhaliwal, Q. He, and E. O. Aboagye, "Early detection of tumor response to chemotherapy by $3^{\prime}$-deoxy- $3^{\prime}-\left[{ }^{18} \mathrm{~F}\right]$ fluorothymidine positron emission tomography: the effect of cisplatin on a fibrosarcoma tumor model in vivo," Cancer Research, vol. 65, no. 10, pp. 4202-4210, 2005.

[3] A. van Waarde, D. C. P. Cobben, A. J. H. Suurmeijer et al., "Selectivity of ${ }^{18} \mathrm{~F}-\mathrm{FLT}$ and ${ }^{18} \mathrm{~F}-\mathrm{FDG}$ for differentiating tumor from inflammation in a rodent model," Journal of Nuclear Medicine, vol. 45, no. 4, pp. 695-700, 2004.

[4] C. Cohade, M. Osman, H. K. Pannu, and R. L. Wahl, "Uptake in supraclavicular area fat ("USA-Fat"): description on ${ }^{18} \mathrm{~F}$ FDG PET/CT," Journal of Nuclear Medicine, vol. 44, no. 2, pp. 170-176, 2003.

[5] A. Debucquoy, E. Devos, P. Vermaelen et al., " ${ }^{18}$ F-FLT and FFFDG PET to measure response to radiotherapy combined with celecoxib in two colorectal xenograft models," International Journal of Radiation Biology, vol. 85, no. 9, pp. 763-771, 2009.

[6] J. R. Bading and A. F. Shields, "Imaging of cell proliferation: status and prospects," Journal of Nuclear Medicine, vol. 49, no. 6, supplement 2, pp. 64S-80S, 2008.
[7] J. S. Rasey, J. R. Grierson, L. W. Wiens, P. D. Kolb, and J. L. Schwartz, "Validation of FLT uptake as a measure of thymidine kinase-1 activity in A549 carcinoma cells," Journal of Nuclear Medicine, vol. 43, no. 9, pp. 1210-1217, 2002.

[8] A. F. Shields, J. R. Grierson, B. M. Dohmen et al., "Imaging proliferation in vivo with [F-18]FLT and positron emission tomography," Nature Medicine, vol. 4, no. 11, pp. 1334-1336, 1998.

[9] S. K. Imam, "Molecular nuclear imaging: the radiopharmaceuticals (review)," Cancer Biotherapy and Radiopharmaceuticals, vol. 20, no. 2, pp. 163-172, 2005.

[10] D. L. Francis, A. Freeman, D. Visvikis et al., "In vivo imaging of cellular proliferation in colorectal cancer using positron emission tomography," Gut, vol. 52, no. 11, pp. 1602-1606, 2003.

[11] H. L. van Westreenen, D. C. P. Cobben, P. L. Jager et al., "Comparison of ${ }^{18}$ F-FLT PET and ${ }^{18} \mathrm{~F}$-FDG PET in esophageal cancer," Journal of Nuclear Medicine, vol. 46, no. 3, pp. 400404, 2005.

[12] A. K. Buck, G. Halter, H. Schirrmeister et al., "Imaging proliferation in lung tumors with PET: ${ }^{18} \mathrm{~F}-\mathrm{FLT}$ versus ${ }^{18} \mathrm{~F}-$ FDG," Journal of Nuclear Medicine, vol. 44, no. 9, pp. 14261431, 2003.

[13] H. Dittmann, B. M. Dohmen, F. Paulsen et al., "[ ${ }^{18}$ F]FLT PET for diagnosis and staging of thoracic tumours," European Journal of Nuclear Medicine and Molecular Imaging, vol. 30, no. 10, pp. 1407-1412, 2003.

[14] W. G. E. Direcks, S. C. Berndsen, N. Proost et al., " ${ }^{18}$ F]FDG and $\left[{ }^{18} \mathrm{~F}\right]$ FLT uptake in human breast cancer cells in relation to the effects of chemotherapy: an in vitro study," British Journal of Cancer, vol. 99, no. 3, pp. 481-487, 2008.

[15] N. Oyama, D. E. Ponde, C. Dence, J. Kim, Y. C. Tai, and M. J. Welch, "Monitoring of therapy in androgen-dependent prostate tumor model by measuring tumor proliferation," Journal of Nuclear Medicine, vol. 45, no. 3, pp. 519-525, 2004.

[16] D. C. Cobben, P. L. Jager, P. H. Elsinga, B. Maas, A. J. H. Suurmeijer, and H. J. Hoekstra, “3/- ${ }^{18} \mathrm{~F}-$ fluoro-3'-deoxy-Lthymidine: a new tracer for staging metastatic melanoma?" Journal of Nuclear Medicine, vol. 44, no. 12, pp. 1927-1932, 2003.

[17] B. Smyczek-Gargya, N. Fersis, H. Dittmann et al., "PET with $\left[{ }^{18} \mathrm{~F}\right]$ fluorothymidine for imaging of primary breast cancer: a pilot study," European Journal of Nuclear Medicine and Molecular Imaging, vol. 31, no. 5, pp. 720-724, 2004.

[18] L. M. Kenny, D. M. Vigushin, A. Al-Nahhas et al., "Quantification of cellular proliferation in tumor and normal tissues of patients with breast cancer by $\left[{ }^{18} \mathrm{~F}\right]$ fluorothymidine-positron emission tomography imaging: evaluation of analytical methods," Cancer Research, vol. 65, no. 21, pp. 10104-10112, 2005.

[19] A. N. Gordon, J. T. Fleagle, D. Guthrie, D. E. Parkin, M. E. Gore, and A. J. Lacave, "Recurrent epithelial ovarian carcinoma: a randomized phase III study of pegylated liposomal doxorubicin versus topotecan," Journal of Clinical Oncology, vol. 19, no. 14, pp. 3312-3322, 2001.

[20] Y. J. Chang, C. H. Chang, C. Y. Yu et al., "Therapeutic efficacy and microSPECT/CT imaging of 188Re-DXR-liposome in a C26 murine colon carcinoma solid tumor model," Nuclear Medicine and Biology, vol. 37, no. 1, pp. 95-104, 2010.

[21] G. D. Luker, V. Sharma, C. M. Pica et al., "Noninvasive imaging of protein-protein interactions in living animals," Proceedings of the National Academy of Sciences of the United States of America, vol. 99, no. 10, pp. 6961-6966, 2002.

[22] C. H. Chang, K. H. Fan, T. J. Chen et al., "Dynamic evaluation of ${ }^{18} \mathrm{~F}-\mathrm{FDG}$ upatke by microPET and whole-body 
autoradiography in a fibrosarcoma-bearing mouse model," Journal of the Formosan Medical Association, vol. 103, no. 11, pp. 876-881, 2004.

[23] D. L. Francis, D. Visvikis, D. C. Costa et al., "Potential impact of $\left[{ }^{18} \mathrm{~F}\right] 3^{\prime}$-deoxy-3' -fluorothymidine versus $\left[{ }^{18} \mathrm{~F}\right]$ fluoro-2deoxy-D-glucose in positron emission tomography for colorectal cancer," European Journal of Nuclear Medicine and Molecular Imaging, vol. 30, no. 7, pp. 988-994, 2003.

[24] K. Ott, K. Herrmann, F. Lordick et al., "Early metabolic response evaluation by fluorine-18 fluorodeoxyglucose positron emission tomography allows in vivo testing of chemosensitivity in gastric cancer: long-term results of a prospective study," Clinical Cancer Research, vol. 14, no. 7, pp. 2012-2018, 2008.

[25] Y. J. Yang, J. S. Ryu, S. Y. Kim et al., "Use of 3'-deoxy$3^{\prime-}\left[{ }^{18} \mathrm{~F}\right]$ fluorothymidine PET to monitor early responses to radiation therapy in murine SCCVII tumors," European Journal of Nuclear Medicine and Molecular Imaging, vol. 33, no. 4, pp. 412-419, 2006.

[26] C. Murayama, N. Harada, T. Kakiuchi et al., "Evaluation of D${ }^{18} \mathrm{~F}-\mathrm{FMT}, 18 \mathrm{~F}-\mathrm{FDG}, \mathrm{L}-11 \mathrm{C}-\mathrm{MET}$, and ${ }^{18} \mathrm{~F}-\mathrm{FLT}$ for monitoring the response of tumors to radiotherapy in mice," Journal of Nuclear Medicine, vol. 50, no. 2, pp. 290-295, 2009.

[27] A. Dimitrakopoulou-Strauss and L. G. Strauss, "The role of ${ }^{18} \mathrm{~F}-\mathrm{FLT}$ in cancer imaging: does it really reflect proliferation?" European Journal of Nuclear Medicine and Molecular Imaging, vol. 35, no. 3, pp. 523-526, 2008.

[28] R. Kameyama, Y. Yamamoto, K. Izuishi et al., "Detection of gastric cancer using ${ }^{18}$ F-FLT PET: comparison with ${ }^{18} \mathrm{~F}$-FDG PET," European Journal of Nuclear Medicine and Molecular Imaging, vol. 36, no. 3, pp. 382-388, 2009.

[29] Y. Yamamotoa, R. Kameyamaa, K. Izuishib et al., "Detection of colorectal cancer using ${ }^{18} \mathrm{~F}$-FLT PET: comparison with ${ }^{18} \mathrm{~F}$ FDG PET," Nuclear Medicine Communications, vol. 30, no. 11, pp. 841-845, 2009. 


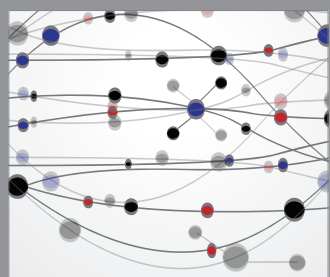

The Scientific World Journal
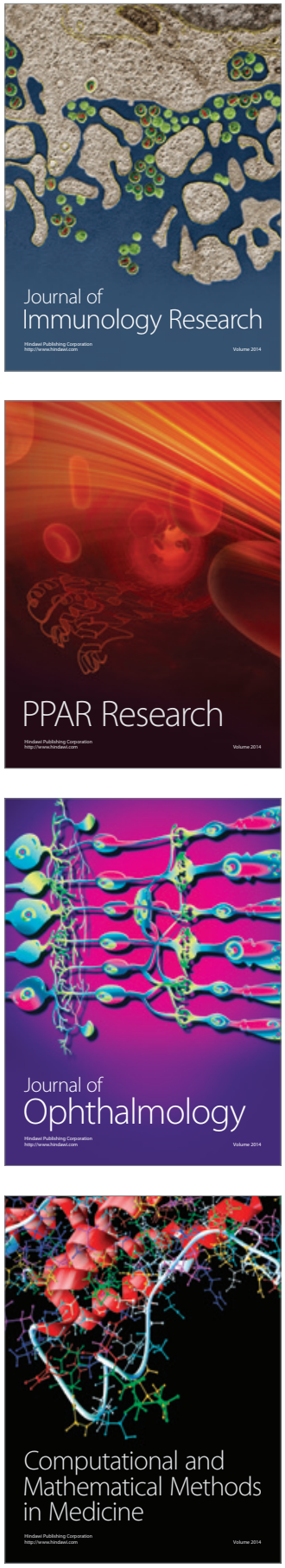

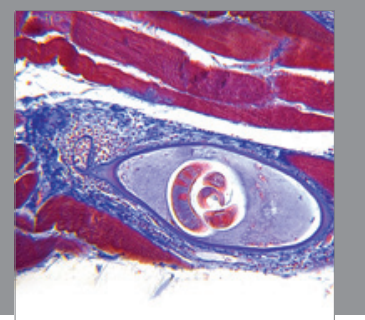

Gastroenterology

Research and Practice
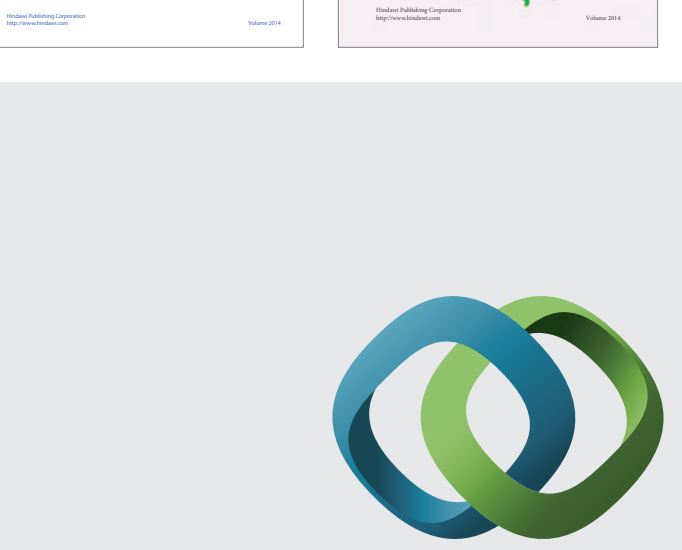

\section{Hindawi}

Submit your manuscripts at

http://www.hindawi.com
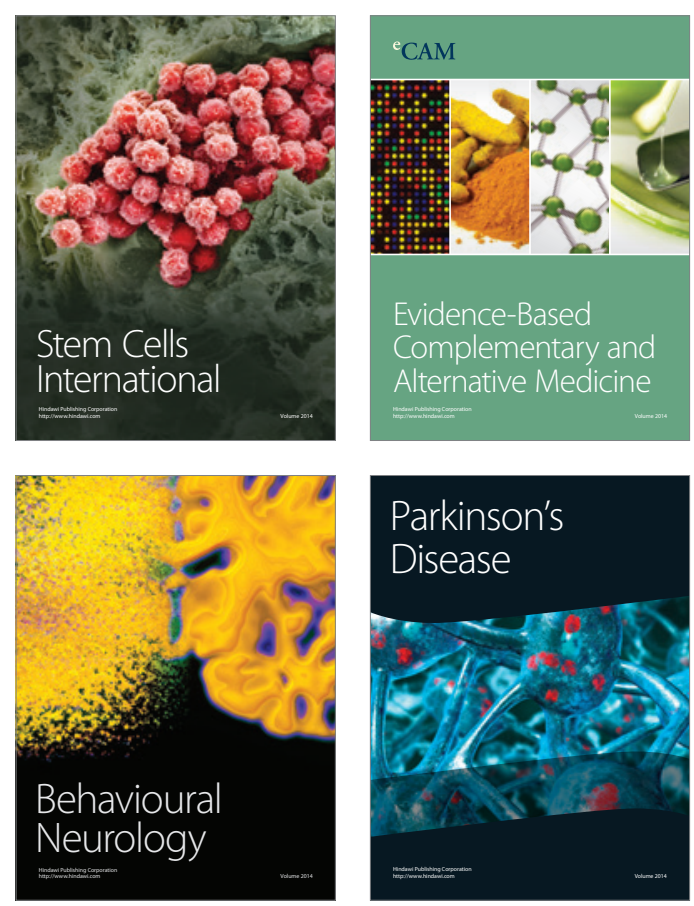

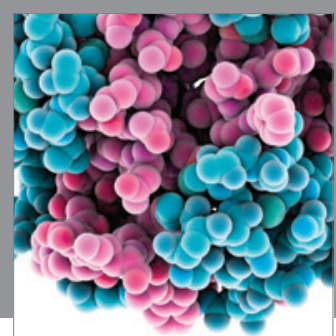

Journal of
Diabetes Research

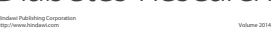

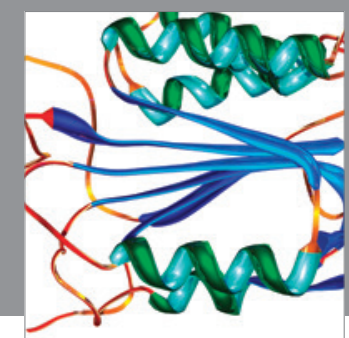

Disease Markers
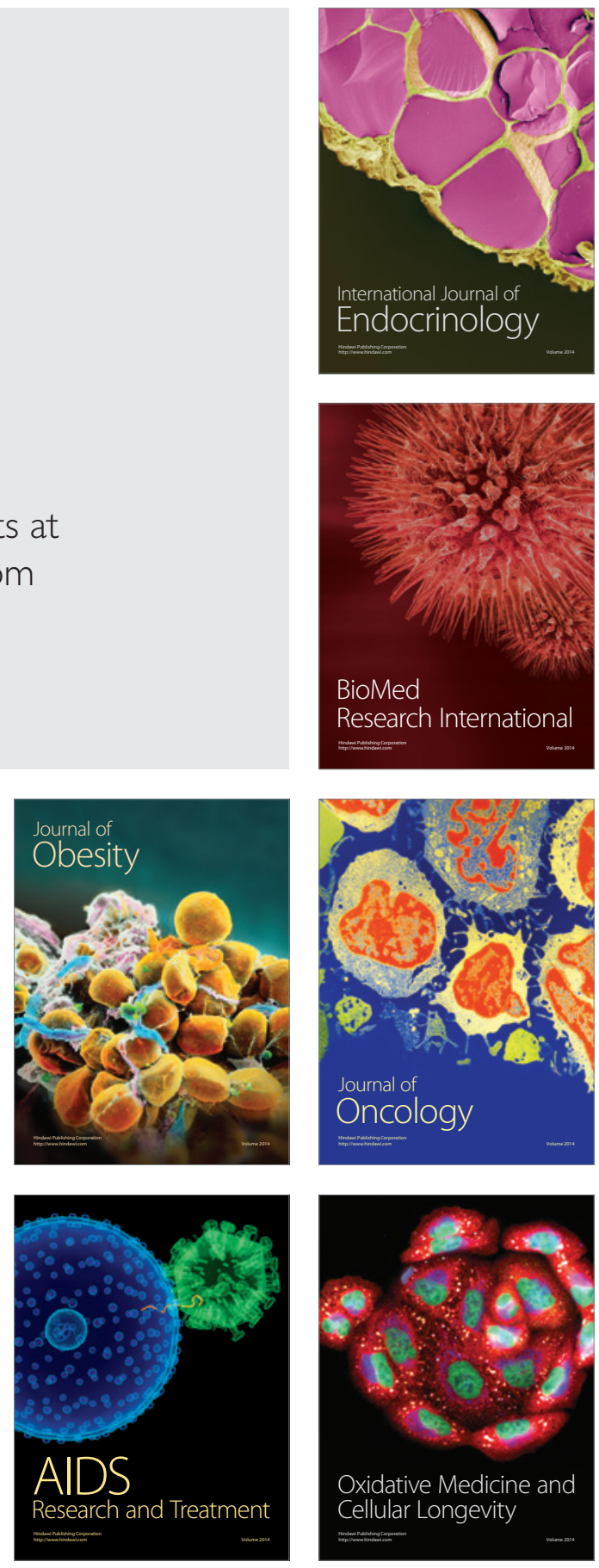